\section{Case Report}

\author{
"Corresponding author \\ Pablo Ruiz-Sada, MD \\ Department of Internal Medicine \\ Hospital Reina Sofía \\ Tudela, Navarra, Spain \\ E-mail: pablitasitas@gmail.com
}

\section{Volume 1 : Issue 1}

Article Ref. \#: 1000IMOJ1103

\section{Article History}

Received: September $8^{\text {th }}, 2016$

Accepted: October $26^{\text {th }}, 2016$

Published: October 26 $6^{\text {th }}, 2016$

\section{Citation}

Ruiz-Sada P, Iguzquiza-Pellejero M-J Esquillor-Rodrigo M-J, Palacios-García L. Cerebellar syndrome as a presentation of pulmonary hypertension. Intern Med Open J. 2016; 1(1): 11-12. doi: 10.17140/IMOJ-1-103

\section{Copyright}

C2016 Ruiz-Sada P. This is an open access article distributed under the Creative Commons Attribution 4.0 International License (CC BY 4.0), which permits unrestricted use, distribution, and reproduction in any medium, provided the original work is properly cited.

\title{
Cerebellar Syndrome as a Presentation of Pulmonary Hypertension
}

\section{Pablo Ruiz-Sada, MD"; María-Jesús Iguzquiza-Pellejero, MD1; María-José Esquillor-Rodrigo, PhD'; Lara Palacios-García, MD²}

${ }^{1}$ Department of Internal Medicine, Hospital Reina Sofía, Tudela, Navarra, Spain

${ }^{2}$ Department of Internal Medicine, Hospital Clínico Universitario de Basurto, Bilbao, Spain

\section{ABSTRACT}

Coincident focal signs and dyspnea can lead to diverse conclusions. However, if they present in the context of a genetic cardiac abnormality a straight forward link can be established. Patent foramen ovale (PFO) can cause a wide range of manifestations. Not always suspected in the Accident and Emergency (A\&E) unit, PFO is the most common cardiac abnormality and should be ruled out when systemic embolisms happen in the absence of cardiac arrhythmia or advanced arteriosclerosis. We present a complex case of a lady in which both PFO and an advanced rheumatic condition were the underlying causes of pulmonary hypertension $(\mathrm{PH})$.

KEYWORDS: Cerebellar; Patent foramen ovale; Dyspnea.

\section{INTRODUCTION}

A 56-year-old woman with a past history of seronegative rheumatoid arthritis treated with leflunomide came to the Accident and Emergency (A\&E) unit complaining of one month of shortness of breath, grade III New-York Heart Association (NYHA), and 48 hours of dysarthria and gait and postural instability causing several falls. Upon physical examination, we found a wide-based gait with negative Romberg test, dysarthria without language impairment and 2 heart murmurs: a systolic one around the tricuspid area and a holosystolic mitral-area localized. Computed-tomography (CT) scan showed a sub-acute infarct in the left superior cerebellarartery region. Laboratory results revealed an acute respiratory failure and an electrocardiogram (ECG) the following findings (Figure 1).

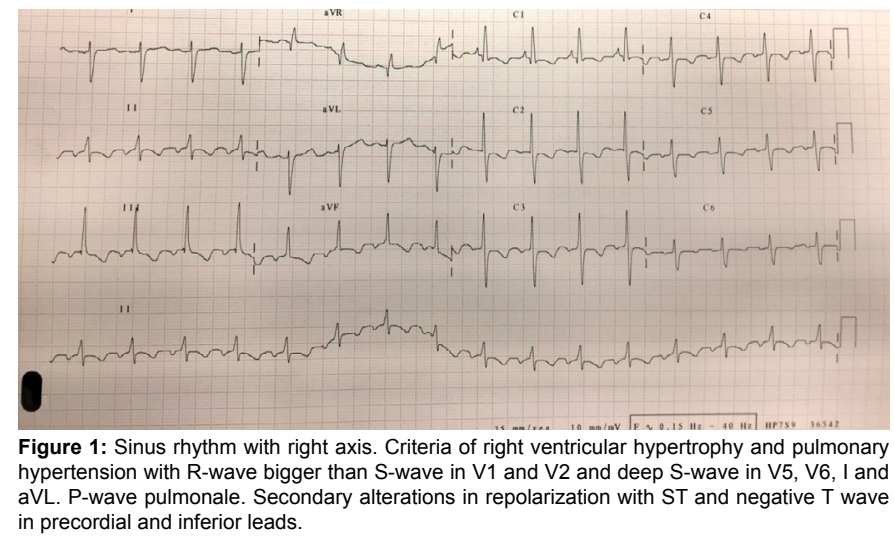

The patient underwent full assessment and an angio-CT scan was performed to rule out pulmonary embolism (PE) and interstitial lung disease (ILD). As well as the angio-CT scan, a supra aortic trunks doppler scan was normal. Transthoracic echocardiogram (TTE), however, estimated a systolic pressure in pulmonary artery of $128 \mathrm{mmHg}$ and the microbubble injection 
technique, the presence of bubbles in the atrium and left ventricle in the $1^{\text {st }} 3$ beats after opacification of the right cavities. These highly suggestive findings of patent foramen ovale (PFO) were confirmed by the right heart catheterization and defined as a PFO type interatrial communication. A lack of response to the acute vasodilatador test with epoprostenol and a right to left communication with a continuous shunt were observed. We closed the anatomic defect and started her on ambrisentan, tadalafil, aspirin and optimized her underlying rheumatic condition treatment.

\section{DISCUSSION}

Pulmonary hypertension ( $\mathrm{PH})$ is a disease characterized by elevated pulmonary artery pressure, which often results in right ventricular failure. It may be idiopathic, familial, or associated with multiple other diseases. PH occurs in men and women of any race or age. $\mathrm{PH}$ can be a progressive, fatal disease if untreated, although the rate of progression is highly variable. ${ }^{1}$ Interestingly, our patient reached an advanced end organ damage stage, but she tailored to her symptoms gradually. After diagnosis, patients with PH should be evaluated in a center with expertise in management of this disease. ${ }^{2}$

Primary or secondary PH classification no longer exists and a new one including 5 groups has been proposed by the World Health Organization (WHO) ${ }^{3} \mathrm{PH}$ in our case caused right ventricular failure (represented in the TTE and ECG findings) and presented as an acute cerebellar syndrome due to a paradoxical cerebral stroke. Both PFO and aortic regurgitation (AR) were the causes of $\mathrm{PH}$ here. As the presence of advanced disease in $\mathrm{PH}$ is generally suggested to be less responsive to therapy, we do not expect in our case a great succes. ${ }^{4}$ A take-home message are the need of a proactive search of right ventricular overload findings in ECG and the clinical suspicion of PFO as the cause of an unexplained cerebral stroke.

\section{CONCLUSIONS}

PFO can cause a wide range of manifestations. Not always suspected in the A\&E unit, we need to expand the awareness of this entity to general and junior doctors because the management and prognosis of the patients rely on a prompt and accurate response.

\section{CONFLICTS OF INTEREST}

The authors declare that they have no conflicts of interest.

\section{REFERENCES}

1. Simonneau G, Gatzoulis MA, Adatia I, et al. Updated clinical classification of pulmonary hypertension. $\mathrm{J} \mathrm{Am} \mathrm{Coll} \mathrm{Cardiol}$. 2013; 62: D34-D41. doi: 10.1016/j.jacc.2013.10.029

2. Stein PD, Matta F, Hughes PG. Scope of problem of pulmonary arterial hypertension. Am J Med. 2015; 128: 844-851. doi: 10.1016/j.amjmed.2015.03.007
3. Galiè N, Corris PA, Frost A, et al. Updated treatment algorithm of pulmonary arterial hypertension. J Am Coll Cardiol. 2013; 62: D60-D72. doi: 10.1016/j.jacc.2013.10.031

4. Badesch DB, Champion HC, Sanchez MA, et al. Diagnosis and assessment of pulmonary arterial hypertension. J Am Coll Cardiol. 2009; 54: S55-S66. doi: 10.1016/j.jacc.2009.04.011 